\title{
The Relationship between Anthropometric Measures and Cardiometabolic Health in Shift Work: Findings from the Atlantic PATH Cohort Study
}

\author{
ABSTRACT \\ Purpose: To evaluate the relationship between anthropometric measures and \\ cardiometabolic health in shift workers compared to non-shift workers.
}

Methods: A population health study with 4,155 shift workers and 8,258 non-shift workers from the Atlantic Partnership for Tomorrow's Health (PATH) cohort. Linear and logistic regression models were used to assess the differences in anthropometric measures (body adiposity) and self-reported cardiometabolic disease outcomes (obesity, diabetes and cardiovascular disease) between shift workers and non-shift workers.

Results: There was a significant increased risk of cardiovascular disease, obesity and diabetes among shift workers compared to matched controls, despite higher levels of physical activity, and lower levels of sedentary behaviour. Shift workers were $17 \%$ more likely to be obese (95\% CI, 7-27) and 27\% more likely to have diabetes (95\% CI, 8-51). The strength of this association was demonstrated by also controlling for body mass index and fat mass index.

Conclusions: Shift work is associated with obesity, cardiovascular disease and diabetes, despite higher levels of physical activity and lower levels of sedentary behaviour. The association between shift work and cardiometabolic health was independent of body mass index for cardiovascular disease and diabetes, and independent of fat mass index for diabetes.

Key Terms: Atlantic PATH, shift work, anthropometric measures, body mass index, fat mass index, cardiometabolic health, diabetes, obesity, cardiovascular disease

Key Messages

- Shift workers experienced a higher risk of diabetes compared to non-shift workers, independent of both body mass index and fat mass index.

- Shift workers had higher rates of cardiovascular disease, obesity and increased fat mass than nonshift workers.

- The higher physical activity and lower sitting time of shift workers did not mitigate these effects. 


\section{Introduction}

Contemporary work environments, particularly healthcare, security and service sectors, increasingly rely upon a 24-hour work cycle to increase productivity, which results in more employees exposed to shift work (Antunes, Levandovski, Dantas, Caumo, \& Hidalgo, 2010; Torquati, Mielke, Brown, \& Kolbe-Alexander, 2018; van Drongelen, Boot, Merkus, Smid, \& van der Beek, 2011; Vetter et al., 2016). Shift work involves a work schedule that is outside of a regular daytime schedule, including evening, night or rotating shifts (Gan et al., 2015; Wang et al., 2012). Thirty percent of working age Canadians work evening, night and rotating shifts (Institute of Health and Work, 2010; Shields, 2002), and 21\% of workers in the European Union (EU) (Eurofound, 2016). Thirteen percent of all Canadians work overnight shifts between 12am5am (CAREX Canada, 2015) and 19\% of workers in the EU perform night work on at least a monthly basis (Eurofound, 2016; Kecklund \& Axelsson, 2016).

Shift work can affect work performance and is associated with disruption to family and social life, stress, anxiety, depression, fatigue, and irregular sleep patterns (Gan et al., 2015; Vogel, Braungardt, Meyer, \& Schneider, 2012; Wyse et al., 2017). Compared to regular daytime shifts, shift work has the potential for disturbing sleep patterns and disrupting circadian rhythms (Boivin \& Boudreau, 2014; Buchvold, Pallesen, Waage, \& Bjorvatn, 2018; Institute of Health and Work, 2010; Torquati et al., 2018; Wyse et al., 2017). Circadian disruption and exposure to light at night can result in both acute and chronic health effects, including cardiovascular, metabolic, reproductive, gastrointestinal, immunological, and neurological outcomes (Buchvold et al., 2018; Depner, Stothard, \& Wright Jr., 2014; Frost, Kolstad, \& Bonde, 2009; Loef et al., 2016; National Toxicology Program, 2016; Puttonen, Härmä, \& Hublin, 2010; Qian \& Scheer, 2016; Torquati et al., 2018; van Drongelen et al., 2011; Vetter et al., 2018; Vetter et al., 2016; Vogel et al., 2012). It is important to consider the relationship between circadian disruption and cardiometabolic health outcomes, such as diabetes, obesity and cardiovascular disease (CVD). Puttonen et al. (Puttonen et al., 2010) demonstrate three distinct, but inter-related, pathways whereby circadian stress results in factors that may influence the development of CVD. Circadian stress produced by shift work encompasses the psychosocial, behavioural and physiological consequences of disturbances to the circadian rhythm. Psychosocial stress includes work stress and work-life balance; behavioural stress may impact sleep quality, smoking, diet, and physical activity; and physiological stress may result in biological mechanisms such as 
increased levels of inflammation and blood pressure. All of these factors are inter-related and may influence other chronic conditions such as obesity and diabetes, as well as the development of CVD (Depner et al., 2014; Puttonen et al., 2010).

We aimed to examine the relationship between anthropometric measures (body adiposity) and cardiometabolic health (CVD, obesity and diabetes) in shift workers using data from the Atlantic Partnership for Tomorrow's Health (Atlantic PATH). The Atlantic PATH study is part of the Canadian Partnership for Tomorrow Project, a pan-Canadian longitudinal cohort study examining the role of genetic, environmental, behavioural, and lifestyle factors in the development of cancer and chronic disease (Dummer et al., 2018; Sweeney et al., 2017).

\section{Methods}

Participants in the Atlantic PATH study were residents of one of the four Atlantic Canadian provinces (Nova Scotia, New Brunswick, Newfoundland and Labrador, and Prince Edward Island). Participants were recruited between 2009-2015, with follow-up planned over a period of 30 years which will support longitudinal research on health impacts of shift work and many other factors, in addition to this cross-sectional research utilizing baseline data. Details on recruitment and data collection have been previously described (Sweeney et al., 2017; Yu, Parker, \& Dummer, 2014). Self-measured anthropometric indices were collected and physical measurements (height, weight, waist and hip circumference, blood pressure, and body composition, including percentage body fat, fat mass and fat free mass) were measured by research nurses using standard operating procedures at an assessment centre. Participants also completed a set of standardized questionnaires on sociodemographic characteristics, employment status, work schedule, health, diet, and lifestyle factors.

While the Atlantic PATH study included participants aged 35-69, we selected participants in the age range of 35-65 to represent the working age population. In total, 20,584 participants provided data on paid work and work schedules, with data from 4,155 shift workers available for inclusion in this study (Figure 1). We conducted a 1:2 match according to participants’ age ( \pm 2 years), sex and education. The final sample consisted of 12,413 participants, including 4,155 shift workers and 8,258 non-shift workers. Fifty-two shift workers were matched with a single non-shift worker as it was not possible to find a second matched participant. Shift workers represent approximately $13 \%$ of the total number of participants in the 
overall Atlantic PATH cohort, and $20 \%$ of the working age participants included in this study (Sweeney et al., 2017).

\section{Assessment of work schedule}

We assessed participants’ shift work exposure based on their response to a question which asked, “[w] hich one of the following best describes your working schedule in your current job? A night shift is work during the early hours of the morning, after midnight. An evening shift is work during the evening ending at or before midnight." Response options included regular daytime schedule or shift, regular evening shift, regular night shift, rotating shift (changing periodically from days to evenings or to nights), split shift (consisting of two or more distinct periods each day), irregular schedule or on call, or other. We have categorized participants as shift workers if they reported working an irregular schedule, including split day shift, evening shift, night shift, rotating shift, or other schedules. We further grouped shift workers as 1) daytime shift workers for those who reported an irregular daytime work schedule; 2) evening/occasional night shift workers (including split shift); and 3) regular night shift workers.

\section{Physical measurements}

Body weight, percentage body fat, fat mass, and fat free mass were measured using the Tanita bioelectrical impedance device (Tanita BC-418, Tanita Corporation of America Inc., Arlington Heights, Illinois). Height was measured by a Seca stadiometer. Body mass index (BMI) was calculated as weight in kilograms divided by height in meters squared. We also calculated fat mass index (FMI) and fat free mass index (FFMI) by dividing fat mass and fat free mass in kilograms by height in meters squared, respectively. A high FMI was defined as $\geq 75$ percentile of age- and sex-specific fat mass distribution as reported by Schutz et al. (Schutz, Kyle, \& Pichard, 2002). We rescaled trunk fat mass, trunk fat free mass, leg fat mass, and leg fat free mass by using the residual method with adjustment for body weight for males and females separately (Cole, Fewtrell, \& Prentice, 2008). Waist and hip circumferences were measured by using Lufin steel tape. Obesity was defined as a BMI $\geq 30 \mathrm{~kg} / \mathrm{m}^{2}$. Abdominal obesity was defined as a waist circumference $\geq 102 \mathrm{~cm}$ for men or $88 \mathrm{~cm}$ for women (Grundy, Cleeman, Daniels, \& et al., 2005).

Observer measured physical data for weight and height were available for $64 \%$ of participants, $60 \%$ for waist and hip circumference, $62 \%$ for body composition, and 36\% for blood pressure. However, participants also reported self-measured weight, height, and waist and 
hip circumference as part of the questionnaire. For those participants who had missing data in observer measured anthropometric measurements, we utilized the self-measured data in the analysis. In all, anthropometric data were available for $91 \%$ of participants in weight and height, and $88 \%$ for waist and hip circumference.

Based on participants' responses to questions on health conditions diagnosed by a physician (yes/no), diabetes mellitus was defined as self-reported diabetes mellitus. CVD was defined as self-reported coronary heart disease or stroke.

\section{Assessment of sociodemographic and behavioural factors}

Due to a lack of ethnic diversity in the cohort, ethnicity was categorized as white and non-white, which is reflective of the larger Atlantic Canadian population (Sweeney et al., 2017). Educational attainment was categorized as high school or less, college level, and university level or higher. Marital status was grouped as married or living together, and single, divorced, separated, or widowed.

For smoking behaviour, participants were grouped as non-smoker, former smoker and current smoker. For alcohol consumption, participants were classified as abstainer, occasional drinker ( $>0$ to $\leq 2-3$ times/month), regular drinker ( $\geq 1$ time/week to $\leq 2-3$ times/week), and habitual drinker ( $\geq 4-5$ times/week).

Physical activity was assessed by using the International Physical Activity Questionnaire (IPAQ). Both the long form and short form questionnaires were completed by participants. To evaluate total activity levels, we calculated metabolic equivalent minutes per week (MET$\mathrm{min} /$ week) for each participant with data derived from the long and short form questionnaires, respectively. The MET scores were computed according to the IPAQ scoring protocol (International Physical Activity Questionnaire (IPAQ), 2016). We then ranked the sex-specific total MET scores into tertiles for data derived categories from the long and short form questionnaires, separately. Levels of total physical activity were classified as low, medium, and high by the low- to high-MET score tertiles. Participants in the highest tertile of the MET score were classified as being physically active. Sitting time was assessed as the self-reported average hours per day spent sitting.

\section{Statistical Analyses}

We performed the statistical analysis using the maximum number of non-missing values available in the matched study sample. We utilized logistic regression models to compute odds 
ratios (OR) and 95\% confidence intervals (CI) for the binary health outcomes selecting non-shift workers as the reference group. These outcomes included a high level of physical activity, obesity, abdominal obesity, high FMI, diabetes, and CVD. In the multivariable regression analysis, we adjusted for province, ethnicity, marital status, smoking and alcohol use. We employed general linear regression models to evaluate the differences in continuous variables between shift workers and non-shift workers, i.e., physical measurements, including anthropometric measures body composition and sitting time per day. We conducted stratified analyses looking at the associations among daytime shift workers, evening/occasional night shift workers, and regular night shift workers. To look at whether body adiposity might mediate the associations between shift work with diabetes and CVD, we adjusted for BMI or FMI in the logistic regression analyses. Statistical significance was defined as $\mathrm{P}<0.05$. All $\mathrm{P}$ values were two-sided. Data management and analyses were performed with SAS statistical package version 9.4 (SAS Institute, Cary, North Carolina).

\section{Results}

\section{Shift Workers v. Non-Shift Workers}

The characteristics of study participants are detailed in Table 1. Consistent with the overrepresentation of women in the larger Atlantic PATH study (69.7\% women and 30.3\% men), women represented 67.2\% of the sample. Most shift workers resided in Nova Scotia (57.7\%), with 26.4\% from New Brunswick, 12.6\% from Newfoundland and Labrador, and 3.3\% from Prince Edward Island.

Physical activity levels were consistently higher among shift workers than non-shift workers. For instance, shift workers were 58\% more likely to report high levels of physical activity compared to non-shift workers (95\% CI, 46-71). Medium levels of physical activity were reported by $28.6 \%$ of shift workers and $33.5 \%$ of non-shift workers, with low levels reported by $29.5 \%$ of shift workers and $35.2 \%$ of non-shift workers (Table 1).

In addition to high levels of physical activity, shift workers had much lower levels of sedentary behaviour with 5.0 hours of sitting time per day (95\% CI, 4.7-5.4) compared to 5.8 hours for non-shift workers (95\% CI, 5.4-6.1) (Table 2). Shift workers had a larger waist circumference (94.6 cms vs. $93.9 \mathrm{cms}, P=0.019)$ and BMI $\left(29.6 \mathrm{~kg} / \mathrm{m}^{2}\right.$ vs. $\left.29.1 \mathrm{~kg} / \mathrm{m}^{2}, P=<0.001\right)$ compared to non-shift workers (Table 2). Overall, shift workers were $17 \%$ more likely to be 
obese (95\% CI, 7-27) (Table 3). We also found that shift workers had a higher FMI of 11.2 $\mathrm{kg} / \mathrm{m}^{2}$ (95\% CI, 10.4-11.9) than non-shift workers with $10.9 \mathrm{~kg} / \mathrm{m}^{2}$ (10.1-11.6) (Table 2).

We further tested whether there were associations between shiftwork and cardiometabolic health. Shift workers were $27 \%$ more likely to have diabetes than non-shift workers (95\% CI, 851), with $5.7 \%$ of shift workers reporting a diagnosis of diabetes mellitus compared to $4.4 \%$ of non-shift workers. Additional analysis revealed that even after controlling for BMI, shift workers were $25 \%$ more likely to have diabetes than non-shift workers (95\% CI, 4-49). This effect remained at 25\% after controlling for FMI (95\% CI, 0-57) (Table 3). Although CVD prevalence was not significant in shift workers versus non-shift workers (31\%; 95\% CI, -1-72), there was an increase after adjustment for BMI (41\%; 95\% CI, 6-88). Adjustment for FMI reduced the effect to $13 \%$ (95\% CI, -22-64) (Table 3), which is consistent with findings that BMI is a better predictor of CVD than measures of adiposity (Ortega, Sui, Lavie, \& Blair, 2016). However, other studies have found BMI and waist circumference in combination to best predict CVD (Mohammadifard et al., 2013) or prioritize other adiposity measures (Iliodromiti et al., 2018).

\section{Health Outcome Findings among Shift Work Categories}

Additional analyses showed differences in anthropometric, body composition, and cardiometabolic disease prevalence between the categories of shift workers, including daytime shift work, evening/occasional night shift work, and regular night shift work compared to nonshift workers (Supplementary Tables 1-2). All three groups of shift workers reported higher levels of physical activity than their non-shift work counterparts. Evening/occasional night shift workers were $142 \%$ more likely to have a high level of physical activity than the reference group (95\% CI, 83-221); regular night shift workers were 78\% more likely (95\% CI, 56-103); and daytime shift workers were 38\% more likely (95\% CI, 25-54).

There were also differences among the groups when considering sedentary behaviour in the form of sitting time. Daytime shift workers reported 5.4 hours per day sitting versus 6.1 hours $(P<0.001)$ for non-shift workers. Evening shift workers $(4.7$ hours versus 5.5 hours, $P$ $<0.001$ ) and regular night shift workers (4.6 hours versus 5.4 hours, $P<0.001$ ) had the least sedentary time, when compared to non-shift workers. There were significant findings of obesity among regular night shift workers who were $21 \%$ more likely to be obese (95\% CI, 5-39) and 16\% more likely to have abdominal obesity than non-shift workers (95\% CI, 1-34), with daytime shift workers being 19\% more likely to be obese (95\% CI, 6-34) (Table 5). 
Day-time shift workers were 27\% more likely to have diabetes (95\% CI, 1-59), and the estimate was relatively stable although not significant after adjustment for BMI. Regular night shift workers were found to have a 63\% increased likelihood of CVD (95\% CI, 3-158). This increased to $70 \%$ after adjustment for BMI (95\% CI, 4-177), and reduced to 50\% after adjustment for FMI (95\% CI, -22-189).

\section{Discussion}

We found that shift workers had a higher risk of obesity and diabetes compared to non-shift workers, independent of BMI and FMI. There was also a higher risk of CVD among shift workers after adjustment for BMI, and this effect was particularly relevant for night shift workers. These findings are both consistent with, and add to, the recently published results from the UK Biobank study which considered whether shiftwork is independently associated with obesity, diabetes, poor sleep, and well-being in a large, general population based cohort study (Wyse et al., 2017). The UK Biobank study reported that shift work in the United Kingdom was associated with multiple indicators of poor health, including obesity and diabetes, despite shift workers reporting higher levels of physical activity (Wyse et al., 2017). Atlantic PATH participants also reported higher levels of physical activity than non-shift workers, which might be attributed to higher levels of occupation-related physical activity, including work that requires standing and other types of physical labour, as well as activity at home pre- or post-shift. This is in contrast to findings from the Canadian Health Measures Survey which found no difference in physical activity levels between shift workers and non-shift workers (Neil-Sztramko, Gotay, Demers, \& Campbell, 2016) and other studies which found lower levels of physical activity of shift workers compared to non-shift workers (Torquati et al., 2018).

Several other studies have found a relationship between CVD and shift work (AsareAnane, Abdul-Latif, Kwaku Ofori, Abdul-Rahman, \& Amanquah, 2015; Brum, Filho, Schnorr, Bottega, \& Rodrigues, 2015; Gu et al., 2015; Pimenta, Kac, Campos e Souza, Rafaela Rocha, Ferreira, Luciana Maria de Barros Almeida, \& Silqueira, Salete Maria de Fátima, 2011; Torquati et al., 2018; Vyas et al., 2012), and after adjusting for BMI, the magnitude of effect (41\%) in our study is concordant with the $24 \%$ increased risk of myocardial infarction and $5 \%$ for stroke reported by Vyas et al in their meta-analysis (Vyas et al., 2012). These authors also found that night shift work was associated with a 41\% (95\% CI, 13-76) increased risk of coronary event (Vyas et al., 2012), which is comparable to our finding of 70\% (95\% CI, 4-177). Further, in our 
study, evening shift workers had the lowest likelihood of reporting CVD -11\% (95\% CI, -59-95) which was also the only shift work category that Vyas et al. found not to have an elevated risk of coronary events 29\% (95\% CI, -31-141) (Vyas et al., 2012).

Research has consistently demonstrated that shift workers are more overweight and obese than those who do not participate in shift work (Brum et al., 2015; Buchvold et al., 2018; Di Lorenzo et al., 2003; Haus et al., 2016; Itani, Kaneita, Murata, Yokoyama, \& Ohida, 2011; Kim et al., 2013; Morikawa et al., 2007; Ostry, Radi, Louie, \& LaMontagne, 2006; Suwazono et al., 2008; Tanaka et al., 2010; Wyse et al., 2017), which was also evident among the Atlantic PATH population, and night shift workers in particular. Increased BMI, obesity and abdominal obesity are associated with a greater risk for chronic disease, including coronary heart disease, stroke, diabetes, and some cancers (Antunes et al., 2010; Smith, Fritschi, Reid, \& Mustard, 2013). Pan et al. (Pan, Schernhammer, Sun, \& Hu, 2011) and Gan et al. (Gan et al., 2015) demonstrated an association between shift work and type 2 diabetes, which is consistent with research that reports an increased risk for diabetes-related mortality with exposure to shift work (Axelsson \& Puttonen, 2012; Karlsson, Alfredsson, Knutsson, Andersson, \& Torén, 2005). In addition to obesity, Atlantic PATH shift workers were also more likely to have diabetes (Gan et al., 2015; Pan et al., 2011; Shariat, Bahri Mohd Tamrin, Daneshjoo, \& Sadeghi, 2015).

This study has a number of strengths. While much of the research on shift work has traditionally focused on nurses and industrial workers, which are often single-sex or industry specific studies, our study contributes to findings on the impact of shift work among the general working population. This study also benefits from a wide geographic scope that included participants from four Canadian provinces, with a corresponding large sample size which comprises both men and women. Furthermore, we used measured height, weight and body fat data for the majority of participants, which meant we were able to adjust for both BMI and FMI and identify an association between body adiposity and cardiometabolic health.

We also acknowledge that the study has a number of limitations. While this research reflects the baseline period of data collection and is thus necessarily cross-sectional, the longterm nature of the Atlantic PATH cohort study will allow us to expand upon this work with our longitudinal research program and across the Canadian Partnership for Tomorrow Project. The volunteer nature of the study cohort means that the Atlantic PATH shift workers represent a smaller percentage (20\%) than the number of Canadians who engage in shift work (30\%). It also 
means that the age and sex structure are not wholly representative of the working population of Atlantic Canada, although it is in the older age group where the effect of longer term work exposures would be anticipated. The age and sex matching undertaken takes account of these differences, and because of the cohort design all comparisons are internal thus limiting the bias associated with case and control design. The definition of shift work can vary between studies which to some extent limits the generalizability and comparability of the findings. However, we used a question based on Canadian national statistics to gather information on work patterns and it is therefore meaningful within a national context and is also comparable with other work. We were also unable to include data on occupation and industry as this data is not currently available, and this analysis was only able to focus on current employment status and not past shift work. Other potential limitations include the reliance on self-reported data. For instance, both type I and type II diabetes were included in categorization of diabetes by self-reported diagnoses and medication use. However, the prevalence of type I diabetes is approximately 9\% in Canada, so this does not greatly impact our analyses (Public Health Agency of Canada, 2017). Physical activity levels were self-reported and not objectively measured with accelerometers, although our findings are consistent with those using measured data (Wyse et al., 2017). The possibility exists that some shift workers may have changed work categories due to a disability accommodation (e.g., a night worker with diabetes moving to an alternative work pattern), which could potentially lead to an underestimate of the effect of shift work on health.

\section{Conclusions}

The finding that shift workers are 25\% more likely to have been diagnosed with diabetes, even after adjustment for BMI or FMI, suggests that shift work has a diabetogenic biological effect over and above that due to obesity. This study adds further evidence to the association of CVD with shift work, and in particular the deleterious effects of night work, as well as highlighting the impact on anthropometric measures. Despite high levels of physical activity and lower levels of sedentary behaviour, shift workers were more likely to have increased rates of diabetes and obesity, which consequently increases the risk of developing other chronic disease. Our future research will aim to confirm the findings of the relationship between shift work and BMI, FMI, diabetes, and CVD in the wider longitudinal Canadian Partnership for Tomorrow Project, and to investigate the potential pathophysiologic mechanisms. More generally, efforts to reduce the detrimental health outcomes associated with shift work should be encouraged. In particular, shift 
work should be recognized as a risk factor in screening programs focused on CVD and diabetes risk.

\section{Ethics Statement}

Informed Consent: Informed consent was obtained from all in all individual participants included in the study.

Ethical approval: All procedures performed in studies involving human participants were in accordance with the ethical standards of the institutional and/or national research committee and with the 1964 Helsinki declaration and its later amendments or comparable ethical standards.

\section{Conflict of Interest Statement}

The authors report no conflict of interest. All authors have full access to all data in the study, take responsibility for the integrity of the data and the accuracy of the data analysis, and give final approval of the version to be submitted.

\section{References}

Antunes, L. C., Levandovski, R., Dantas, G., Caumo, W., \& Hidalgo, M. P. (2010). Obesity and shift work: Chronobiological aspects. Nutrition Research Reviews, 23(1), 155-168. doi:10.1017/S0954422410000016 [doi]

Asare-Anane, H., Abdul-Latif, A., Kwaku Ofori, E., Abdul-Rahman, M., \& Amanquah, S. (2015). Shift work and the risk of cardiovascular disease among workers in cocoa company, Tema. BMC Research Notes, 8 doi:10.1186/s13104-015-1750-3

Axelsson, J., \& Puttonen, S. (2012). Night shift work increases the risk for type 2 diabetes. Evidence-Based Medicine, 17(6), 193-194. doi:10.1136/ebmed-2012-100649 [doi]

Boivin, D. B., \& Boudreau, P. (2014). Impacts of shift work on sleep and circadian rhythms. Pathologie Biologie, 62(5), 292. doi:http://dx.doi.org/10.1016/j.patbio.2014.08.001 
Brum, M. C. B., Filho, F. F. D., Schnorr, C. C., Bottega, G. B., \& Rodrigues, T. C. (2015). Shift work and its association with metabolic disorders. Diabetology \& Metabolic Syndrome, $7(45)$

Buchvold, H., Pallesen, S., Waage, S., \& Bjorvatn, B. (2018). Shift work schedule and night work load: Effects on body mass index - a four-year longitudinal study. Scandinavian Journal of Work, Environment and Health.

CAREX Canada. (2015). Nova scotia carcinogenic exposures. Burnaby, BC: CAREX Canada.

Cole, T. J., Fewtrell, M. S., \& Prentice, A. (2008). The fallacy of using percentage body fat as a measure of adiposity. Am J Clin Nutr., 87(6), 1959.

Depner, C., Stothard, E., \& Wright Jr., K. (2014). Metabolic consequences of sleep and circadian disorders. Curr Diab Rep, 14, 507. doi:10.1007/s11892-014-0507-z

Di Lorenzo, L., De Pergola, G., Zocchetti, C., L'Abbate, N., Basso, A., Pannacciulli, N., ... Soleo, L. (2003). Effect of shift work on body mass index: Results of a study performed in 319 glucose-tolerant men working in a southern italian industry. International Journal of Obesity and Related Metabolic Disorders : Journal of the International Association for the Study of Obesity, 27(11), 1353-1358. doi:10.1038/sj.ijo.0802419 [doi]

Dummer, T. J. B., Awadalla, P., Boileau, C., Craig, C., Fortier, I., Goel, V., ... CPTP Regional Cohort Committee Group Consortium. (2018). The Canadian Partnership for Tomorrow Project: A pan-Canadian platform for chronic disease prevention research. CMAJ. 
Eurofound. (2016). Sixth european working conditions survey - overview report. Luxembourg: Publications Office of the European Union.

Frost, P., Kolstad, H. A., \& Bonde, J. P. (2009). Shift work and the risk of ischemic heart disease - a systematic review of the epidemiologic evidence. Scand J Work Environ Health, 35(3), 163. doi:10.5271/sjweh.1319

Gan, Y., Yang, C., Tong, X., Sun, H., Cong, Y., Yin, X., . . Lu, Z. (2015). Shift work and diabetes mellitus: A meta-analysis of observational studies. Occupational and Environmental Medicine, 72(1), 72-78. doi:10.1136/oemed-2014-102150 [doi]

Grundy, S. M., Cleeman, J. I., Daniels, S. R., \& et al. (2005). Diagnosis and management of the metabolic syndrome an american heart association/national heart, lung, and blood institute scientific statement. Circulation, 112(17), 2735. doi:0.1161/CIRCULATIONAHA.105.169404

Gu, F., Han, J., Laden, F., Pan, A., Caporaso, N. E., Stampfer, M. J., .. Schernhammer, E. S. (2015). Total and cause-specific mortality of U.S. nurses working rotating night shifts. American Journal of Preventive Medicine, 48(3), 241-252. doi:10.1016/j.amepre.2014.10.018 [doi]

Haus, E., Reinberg, A., Mauvieux, B., Le Floc'h, N., Sackett-Lundeen, L., \& Touitou, Y. (2016). Risk of obesity in male shift workers: A chronophysiological approach. Chronobiology International, 33(8), 1018-1036. doi:10.3109/07420528.2016.1167079 [doi] 
Iliodromiti, S., Celis-Morales, C., Lyall, D., Anderson, J., Gray, S., MacKay, D., ... Sattar, N. (2018). The impact of confounding on the associations of different adiposity measures with the incidence of cardiovascular disease: A cohort study of 296,535 adults of white European descent. European Heart Journal, 39

Institute of Health and Work. (2010). The prevalence of shift work in Canada. Retrieved from https://www.iwh.on.ca/shift-work-symposium/demers

International Physical Activity Questionnaire (IPAQ). (2016). IPAQ scoring protocol. Retrieved from https://sites.google.com/site/theipaq/scoring-protoco

Itani, O., Kaneita, Y., Murata, A., Yokoyama, E., \& Ohida, T. (2011). Association of onset of obesity with sleep duration and shift work among japanese adults. Sleep Medicine, 12(4), 341-345. doi:10.1016/j.sleep.2010.09.007 [doi]

Karlsson, B., Alfredsson, L., Knutsson, A., Andersson, E., \& Torén, K. (2005). Total mortality and cause-specific mortality of Swedish shift- and dayworkers in the pulp and paper industry in 1952-2001. Scandinavian Journal of Work, Environment and Health, 31(1), 30.

Kecklund, G., \& Axelsson, J. (2016). Health consequences of shift work and insufficient sleep. BMJ, 355 doi:10.1136/bmj.i5210

Kim, M. J., Son, K. H., Park, H. Y., Choi, D. J., Yoon, C. H., Lee, H. Y., . . Cho, M. C. (2013). Association between shift work and obesity among female nurses: Korean nurses' 
survey. BMC Public Health, 13, 1204-2458-13-1204. doi:10.1186/1471-2458-13-1204 [doi]

Loef, B., van Baarle, D., van der Beek, A. J., van Kerkhof, L. W., van de Langenberg, D., \& Proper, K. I. (2016). Klokwerk + study protocol: An observational study to the effects of night-shift work on body weight and infection susceptibility and the mechanisms underlying these health effects. BMC Public Health, 16, 692-016-3317-1. doi:10.1186/s12889-016-3317-1 [doi]

Mohammadifard, N., Nazem, M., Sarrafzadegan, N., Nouri, F., Sajjadi, F., Maghroun, M., \& Alikhasi, H. (2013). Body mass index, waist-circumference and cardiovascular disease risk factos in iranian adults: Isfahan healthy heart program. Journal of Health, Population and Nutrition, 31(3)

Morikawa, Y., Nakagawa, H., Miura, K., Soyama, Y., Ishizaki, M., Kido, T., ... Suwazon, Y., Nogawa, K. (2007). Effect of shift work on body mass index and metabolic parameters . Scand J Work Environ Health, 33(1), 45.

National Toxicology Program. (2016). Shift work at night, artificial light at night, and circadian disruption workshop. Office of the Report on Carcinogens, Office of Health Assessment and Translation, Division of the National Toxicology Program, National Institute of Environmental Health Services, U.S. Department of Health and Human Services.

Neil-Sztramko, S. E., Gotay, C. C., Demers, P. A., \& Campbell, K. L. (2016). Physical activity, physical fitness, and body composition of Canadian shift workers: Data from the 
Canadian health measures survey cycles 1 and 2. Journal of Occupational and Environmental Medicine, 58(1), 94-100. doi:10.1097/JOM.0000000000000574 [doi]

Ortega, F., Sui, X., Lavie, C., \& Blair, S. (2016). Body mass index, the most widely used but also widely criticized index: Would a gold-standard measure of total body fat be a better predictor of cardiovascular disease mortality? Mayo Clinic Proceedings, 91(4), 443. doi:10.1016/j.mayocp.2016.01.008

Ostry, A., Radi, S., Louie, A., \& LaMontagne, A. (2006). Psychosocial and other working conditions in relation to body mass index in a representative sample of Australian workers. BMC Public Health, 6(53)

Pan, A., Schernhammer, E. S., Sun, Q., \& Hu, F. B. (2011). Rotating night shift work and risk of type 2 diabetes: Two prospective cohort studies in women. PLoS Medicine, 8(12), e1001141. doi:10.1371/journal.pmed.1001141 [doi]

Pimenta, A. M., Kac, G., Campos e Souza, Rafaela Rocha, Ferreira, Luciana Maria de Barros Almeida, \& Silqueira, Salete Maria de Fátima. (2011). Night-shift work and cardiovascular risk among employees of a public university. Rev Assoc Med Bras, 58(2), 168.

Public Health Agency of Canada. (2017). Diabetes in canada. Ottawa, Ontario: Public Health Agency of Canada. 
Puttonen, S., Härmä, M., \& Hublin, C. (2010). Shift work and cardiovascular disease pathways from circadian stress to morbidity. Scand J Work Environ Health, 36(2), 96. doi:10.5271/sjweh.2894

Qian, J., \& Scheer, F. (2016). Circadian system and glucose metabolism: Implications for physiology and disease. Trends Endocrinol Metab, 27(5), 282. doi:10.1016/j.tem.2016.03.005

Schutz, Y., Kyle, U. U. G., \& Pichard, C. (2002). Fat-free mass index and fat mass index percentiles in caucasians aged 18-98 y. Int J Obes Relat Metab Disord., 26(7) doi:10.1038/sj.ijo.0802037

Shariat, A., Bahri Mohd Tamrin, S., Daneshjoo, A., \& Sadeghi, H. (2015). The adverse health effects of shift work in relation to risk of illness/disease: A review. Acta Medica Bulgarica, XLII(1)

Shields, M. (2002). Shift work and health. Health Reports, 13(4), 11-33.

Smith, P., Fritschi, L., Reid, A., \& Mustard, C. (2013). The relationship between shift work and body mass index among Canadian nurses. Applied Nursing Research : ANR, 26(1), 24-31. doi:10.1016/j.apnr.2012.10.001 [doi]

Suwazono, Y., Dochi, M., Sakata, K., Okubo, Y., Oishi, M., Tanaka, K., . . Nogawa, K. (2008). A longitudinal study on the effect of shift work on weight gain in male Japanese workers. Obesity (Silver Spring, Md.), 16(8), 1887-1893. doi:10.1038/oby.2008.298 [doi] 
Sweeney, E., Cui, Y., DeClercq, V., Devichand, P., Forbes, C., Grandy, S., ... Dummer, T. J. B. (2017). Cohort profile: The Atlantic Partnership for Tomorrow's Health (Atlantic PATH) study. International Journal of Epidemiology

Tanaka, K., Sakata, K., Oishi, M., Morimoto, H., Nakada, S., Uetani, M., ... Suwazono Yasushi. (2010). Estimation of the benchmark duration of shiftwork associated with weight gain in male Japanese workers. Chronobiology International, 27(9-10), 1895.

Torquati, L., Mielke, G. I., Brown, W. J., \& Kolbe-Alexander, T. (2018). Shift work and the risk of cardiovascular disease. A systematic review and meta-analysis including doseresponse relationship. Scand J Work Environ Health, 44(3), 229.

doi:10.5271/sjweh.3700

van Drongelen, A., Boot, C., Merkus, S., Smid, T., \& van der Beek, A. (2011). The effects of shift work on body weight change - a systematic review of longitudinal studies. Scand J Work Environ Health, 37(4), 263. doi:10.5271/sjweh.3143

Vetter, C., Dashti, H., Lane, J., Anderson, S., Schernhammer, E., Rutter, M., . . Scheer, F. (2018). Night shift work, genetic risk, and type 2 diabetes in the UK Biobank diabetes care. Diabetes Care, 41, 762. doi:https://doi.org/10.2337/dc17-1933

Vetter, C., Devore, E. E., Wegrzyn, L. R., Massa, J., Speizer, F. E., Kawachi, I., . . . Schernhammer, E. S. (2016). Association between rotating night shift work and risk of coronary heart disease among women. JAMA, 315(16), 1726-1734. doi:10.1001/jama.2016.4454 [doi] 
Vogel, M., Braungardt, T., Meyer, W., \& Schneider, W. (2012). The effects of shift work on physical and mental health. Journal of Neural Transmission (Vienna, Austria : 1996), 119(10), 1121-1132. doi:10.1007/s00702-012-0800-4 [doi]

Vyas, M., Garg, A., VIansavichus, A., Costella, J., Donner, A., Laugsand, L., ... Hackam, D. (2012). Shift work and vascular events: Systematic review and meta-analysis $B M J, 345(\mathrm{e} 4800)$

Wang, X. S., Travis, R. C., Reeves, G., Green, J., Allen, N. E., Key, T. J., . . Beral, V. (2012). Characteristics of the Million Women Study participants who have and have not worked at night. Scandinavian Journal of Work, Environment \& Health, 38(6), 590-599. doi:10.5271/sjweh.3313 [doi]

Wyse, C., Morales, C., Graham, N., Yu, F., Ward, J., Curtis, A., . . Pell, J. P. (2017). Adverse metabolic and mental health outcomes associated with shiftwork in a populationbased study of 277168 workers in UK Biobank. Annals of Medicine, doi:10.1080/07853890.2017.1292045http://www.refworks.com/refworks2/default.a spx?r=references|MainLayout::init\#

Yu, Z. M., Parker, L., \& Dummer, T. J. B. (2014). Depressive symptoms, diet quality, physical activity, and body composition among populations in Nova Scotia, Canada: Report from the Atlantic Partnership for Tomorrow's Health. Preventive Medicine 61, 106. 


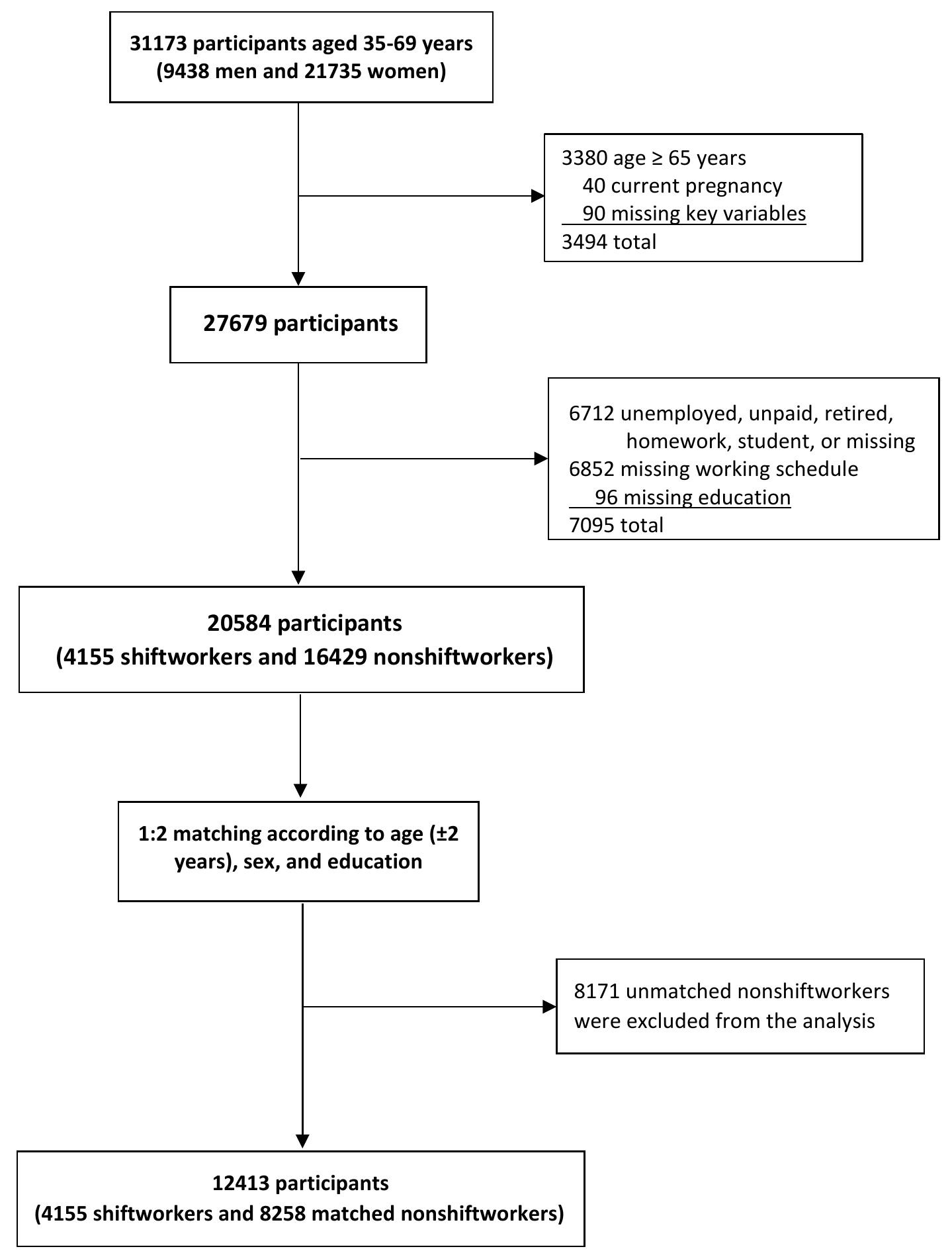


Figure 1. Flow chart of study participant recruitment ${ }^{\mathrm{a}}$

a 52 shiftworkers were matched with only 1 nonshiftworker.

Table 1. Characteristics of study participants

\begin{tabular}{|c|c|c|c|c|c|c|}
\hline \multirow{2}{*}{$\begin{array}{l}\text { Characteristics } \\
\text { Age, year, mean (SD) }\end{array}$} & \multirow{2}{*}{$\begin{array}{c}\mathrm{n}=\mathbf{8 2 5 3} \\
8253\end{array}$} & \multicolumn{2}{|c|}{ Non-shift worker } & \multirow{2}{*}{$\begin{array}{c}n=4155 \\
4155\end{array}$} & \multicolumn{2}{|c|}{ Shift worker } \\
\hline & & 50.4 & $(8.0)$ & & 50.4 & $(8.0)$ \\
\hline Sex, female, $\mathrm{n}(\%)$ & & 5556 & $(67.3)$ & & 2780 & $(66.9)$ \\
\hline \multicolumn{7}{|l|}{ Education, $\mathbf{n}(\%)$} \\
\hline Less than high school & & 1629 & $(19.7)$ & & 843 & $(20.3)$ \\
\hline College level & & 4004 & $(48.5)$ & & 2002 & $(48.2)$ \\
\hline University level & & 1617 & $(19.6)$ & & 869 & $(20.9)$ \\
\hline Postgraduate level & & 1003 & $(12.2)$ & & 441 & $(10.6)$ \\
\hline \multicolumn{7}{|l|}{ Anthropometric Measures } \\
\hline Body weight, kg, mean (SD) & 7573 & 79.7 & $(18.2)$ & 3690 & 80.6 & $(19.1)$ \\
\hline Body height, $\mathrm{cm}$, mean (SD) & 7612 & 167.0 & $(8.1)$ & 3713 & 167.2 & $(8.3)$ \\
\hline Body mass index, $\mathrm{kg} / \mathrm{m}^{2}$, mean (SD) & 7557 & 28.5 & $(6.0)$ & 3679 & 28.8 & $(6.4)$ \\
\hline Waist circumference, cm, mean (SD) & 7337 & 94.1 & $(14.9)$ & 3559 & 94.7 & $(15.2)$ \\
\hline Hip circumference, cm, mean (SD) & 7335 & 105.4 & $(12.5)$ & 3551 & 105.7 & $(12.6)$ \\
\hline Waist-to-hip ratio, mean (SD) & 7313 & 0.89 & $(0.11)$ & 3540 & 0.90 & $(0.10)$ \\
\hline Percentage fat mass, \%, mean (SD) & 4848 & 33.5 & $(9.1)$ & 2470 & 33.5 & $(9.4)$ \\
\hline Fat mass index, $\mathrm{kg} / \mathrm{m}^{2}$, mean (SD) & 4846 & 9.8 & $(4.3)$ & 2467 & 9.9 & $(4.7)$ \\
\hline Fat free mass index, $\mathrm{kg} / \mathrm{m}^{2}$, mean (SD) & 4845 & 18.5 & $(3.2)$ & 2468 & 18.5 & $(3.2)$ \\
\hline Leg fat mass, kg, mean (SD) & 4836 & 9.8 & $(3.5)$ & 2467 & 9.9 & $(3.6)$ \\
\hline Leg fat free mass, $\mathrm{kg}$, mean (SD) & 4839 & 17.3 & $(2.5)$ & 2469 & 17.3 & $(2.7)$ \\
\hline Trunk fat mass, kg, mean (SD) & 4812 & 14.2 & $(3.1)$ & 2450 & 14.2 & $(3.1)$ \\
\hline Trunk fat free mass, kg, mean (SD) & 4810 & 28.7 & $(4.1)$ & 2451 & 28.7 & $(4.3)$ \\
\hline \multicolumn{7}{|l|}{ Province, $\mathrm{n}(\%)$} \\
\hline Nova Scotia & & 3966 & $(48.1)$ & & 2396 & $(57.7)$ \\
\hline New Brunswick & & 2675 & $(32.4)$ & & 1096 & $(26.4)$ \\
\hline Newfoundland and Labrador & & 1278 & $(15.5)$ & & 525 & $(12.6)$ \\
\hline Prince Edward Island & & 334 & $(4.0)$ & & 138 & $(3.3)$ \\
\hline
\end{tabular}




\begin{tabular}{|c|c|c|c|}
\hline White & $7154 \quad(86.7)$ & 3608 & $(86.8)$ \\
\hline Non-white & $559 \quad(6.8)$ & 312 & $(7.5)$ \\
\hline DNK/PNA & $540 \quad(6.5)$ & 235 & $(5.7)$ \\
\hline \multicolumn{4}{|l|}{ Marital status, n (\%) } \\
\hline Married or living together & $6586 \quad(79.8)$ & 3187 & (76.7) \\
\hline Single, divorced, or widowed & $1642(19.9)$ & 949 & $(22.8)$ \\
\hline DNK/PNA & $25 \quad(0.3)$ & 19 & $(0.5)$ \\
\hline Married or living together & $6586 \quad(79.8)$ & 3187 & $(76.7)$ \\
\hline \multicolumn{4}{|l|}{ Smoking status, $\mathrm{n}(\%)$} \\
\hline Never & $4038 \quad(48.9)$ & 2044 & $(49.2)$ \\
\hline Former & $3226 \quad$ (39.1) & 1568 & $(37.7)$ \\
\hline Current & $930 \quad(11.3)$ & 514 & $(12.4)$ \\
\hline DNK/PNA & $59 \quad(0.7)$ & 29 & $(0.7)$ \\
\hline \multicolumn{4}{|l|}{ Total physical activity, $\mathbf{n}(\%)$} \\
\hline Low & $2899 \quad(35.2)$ & 1221 & $(29.5)$ \\
\hline Medium & $2758 \quad(33.5)$ & 1183 & $(28.6)$ \\
\hline High & $2583 \quad(31.3)$ & 1737 & $(41.9)$ \\
\hline \multicolumn{4}{|l|}{ Alcohol drinking, $\mathbf{n}(\%)$} \\
\hline Abstainer & $833 \quad(10.1)$ & 515 & $(12.4)$ \\
\hline Occasional drinker ( $>0$ to $\leq 2-3$ times/month) & $3427(41.5)$ & 1862 & $(44.8)$ \\
\hline Regular drinker ( $\geq 1$ time/week to $\leq 2-3$ times/week) & $2772 \quad(33.6)$ & 1159 & $(27.9)$ \\
\hline Habitual drinker ( $\geq 4-5$ times/week) & $1182(14.3)$ & 600 & $(14.4)$ \\
\hline DNK/PNA & $39 \quad(0.5)$ & 19 & $(0.5)$ \\
\hline Obesity ${ }^{b}$, yes, n (\%) & $2562 \quad(33.9)$ & 1337 & $(36.3)$ \\
\hline Abdominal obesity ${ }^{c}$, yes, $\mathbf{n}(\%)$ & $3704 \quad(50.5)$ & 1848 & $(51.9)$ \\
\hline Diabetes mellitus ${ }^{\mathrm{d}}$, yes, $\mathbf{n}(\%)$ & $363(4.4)$ & 237 & $(5.7)$ \\
\hline Cardiovascular disease ${ }^{\mathrm{e}}$, yes, $\mathrm{n}(\%)$ & $134 \quad(1.6)$ & 89 & $(2.1)$ \\
\hline
\end{tabular}

DNK/PNA, do not know or prefer not to answer

a Data are means (standard deviation) and number of participants (percentage). Percentages may not total 100 due to rounding

${ }^{\mathrm{b}} \mathrm{BMI} \geq 30 \mathrm{~kg} / \mathrm{m}^{2}$

'Waist circumference $\geq 102 \mathrm{~cm}$ for men and $\geq 88 \mathrm{~cm}$ for women

${ }^{\mathrm{d}}$ Self-reported diabetes mellitus

e Self-reported coronary heart disease or stroke 
Table 2. Differences in anthropometric indices,body composition and sedentary behaviour between matched non-shift workers and shift workers

\begin{tabular}{|c|c|c|c|c|c|c|c|}
\hline \multirow{3}{*}{ Body mass index, $\mathrm{kg} / \mathrm{m}^{2}$} & \multicolumn{3}{|c|}{ Non-shift worker } & \multicolumn{3}{|c|}{ Shift worker } & \\
\hline & \multirow[t]{2}{*}{$\mathbf{n}$} & \multicolumn{2}{|r|}{ Mean $(95 \% \mathrm{Cl})$} & \multirow[t]{2}{*}{$\mathbf{n}$} & \multicolumn{2}{|c|}{ Mean $(95 \% \mathrm{Cl})$} & \\
\hline & & & & & & & $P$ value \\
\hline Unadjusted & 7557 & 28.5 & $(28.4-28.7)$ & 3679 & 28.8 & $(28.57-28.97)$ & 0.052 \\
\hline Multivariable adjusted $^{a}$ & & 29.1 & $(28.4-29.9)$ & & 29.6 & $(28.80-30.30)$ & $<0.001$ \\
\hline \multicolumn{8}{|l|}{ Waist circumference, cm } \\
\hline Unadjusted & 7337 & 94.1 & $(93.8-94.4)$ & 3559 & 94.7 & $(94.2-95.2)$ & 0.044 \\
\hline Multivariable adjusted & & 93.9 & (91.9-95.9) & & 94.6 & $(92.6-96.6)$ & 0.019 \\
\hline \multicolumn{8}{|l|}{ Waist-to-hip ratio } \\
\hline Unadjusted & 7313 & 0.89 & $(0.89-0.89)$ & 3540 & 0.90 & $(0.89-0.90)$ & 0.197 \\
\hline Multivariable adjusted & & 0.90 & $(0.88-0.91)$ & & 0.90 & $(0.89-0.92)$ & 0.070 \\
\hline \multicolumn{8}{|l|}{ Percentage fat mass, \% } \\
\hline Unadjusted & 4848 & 33.5 & $(33.3-33.8)$ & 2470 & 33.5 & $(33.1-33.9)$ & 0.897 \\
\hline Multivariable adjusted & & 36.0 & $(34.4-37.5)$ & & 36.3 & $(34.8-37.9)$ & 0.122 \\
\hline \multicolumn{8}{|l|}{ Fat mass index, $\mathrm{kg} / \mathrm{m}^{2}$} \\
\hline Unadjusted & 4846 & 9.8 & $(9.6-9.9)$ & 2467 & 9.9 & $(9.7-10.1)$ & 0.293 \\
\hline Multivariable adjusted & & 10.9 & $(10.1-11.6)$ & & 11.2 & $(10.4-11.9)$ & 0.004 \\
\hline \multicolumn{8}{|l|}{ Fat free mass index, $\mathrm{kg} / \mathrm{m}^{2}$} \\
\hline Unadjusted & 4845 & 18.5 & $(18.4-18.6)$ & 2468 & 18.5 & $(18.4-18.7)$ & 0.382 \\
\hline Multivariable adjusted ${ }^{\mathrm{a}}$ & & 18.5 & $(17.9-19.0)$ & & 18.7 & $(18.1-19.2)$ & 0.009 \\
\hline \multicolumn{8}{|c|}{ Body weight adjusted leg fat mass, kg } \\
\hline Unadjusted & 4836 & 9.8 & $(9.7-9.9)$ & 2467 & 9.9 & $(9.7-10.0)$ & 0.232 \\
\hline Multivariable adjusted & & 10.4 & $(9.8-11.0)$ & & 10.5 & $(9.9-11.1)$ & 0.229 \\
\hline \multicolumn{8}{|c|}{ Body weight adjusted leg fat free mass, kg } \\
\hline Unadjusted & 4839 & 17.3 & $(17.3-17.4)$ & 2469 & 17.3 & $(17.2-17.4)$ & 0.680 \\
\hline Multivariable adjusted & & 16.8 & $(16.3-17.2)$ & & 16.7 & $(16.3-17.2)$ & 0.533 \\
\hline \multicolumn{8}{|c|}{ Body weight adjusted trunk fat mass, kg } \\
\hline Unadjusted & 4812 & 14.2 & $(14.1-14.3)$ & 2450 & 14.2 & $(14.1-14.3)$ & 0.551 \\
\hline Multivariable adjusted & & 14.7 & $(14.2-15.2)$ & & 14.7 & $(14.2-15.3)$ & 0.864 \\
\hline \multicolumn{8}{|c|}{ Body weight adjusted trunk fat free mass, kg } \\
\hline Unadjusted & 4810 & 28.7 & $(28.6-28.8)$ & 2451 & 28.7 & $(28.5-28.8)$ & 0.591 \\
\hline Multivariable adjusted ${ }^{\mathrm{a}}$ & & 27.7 & $(26.9-28.4)$ & & 27.6 & $(26.9-28.3)$ & 0.488 \\
\hline \multicolumn{8}{|l|}{ Sitting time, hour/day } \\
\hline Unadjusted & 7768 & 6.0 & $(5.9-6.0)$ & 3729 & 5.3 & $(5.2-5.3)$ & $<0.001$ \\
\hline Multivariable adjusted $^{\mathrm{a}}$ & & 5.8 & $(5.4-6.1)$ & & 5.0 & $(4.7-5.4)$ & $<0.001$ \\
\hline
\end{tabular}


Data are means (95\% confidence intervals)

${ }^{\text {a }}$ Adjusted for province, ethnicity, marital status, smoking and alcohol use 
Table 3. Associations of shiftwork with physical activity, obesity and cardiometabolic diseases

\begin{tabular}{|c|c|c|c|c|c|}
\hline & \multicolumn{2}{|c|}{ Non-shift worker } & \multicolumn{3}{|c|}{ Shift worker } \\
\hline & Cases/n & OR (95\% CI) & Cases/n & & OR $(95 \% \mathrm{Cl})$ \\
\hline \multicolumn{6}{|l|}{ Physically active $^{\mathrm{b}}$} \\
\hline Unadjusted & $2583 / 8240$ & 1.0 (Reference) & $1737 / 4141$ & 1.58 & $(1.46-1.71)$ \\
\hline Multivariable adjusted & & 1.0 (Reference) & & 1.58 & $(1.46-1.71)$ \\
\hline \multicolumn{6}{|l|}{$\begin{array}{l}\text { Obesity } \\
\end{array}$} \\
\hline Unadjusted & $2562 / 7557$ & 1.0 (Reference) & $1337 / 3679$ & 1.11 & $(1.03-1.21)$ \\
\hline Multivariable adjusted & & 1.0 (Reference) & & 1.17 & $(1.07-1.27)$ \\
\hline \multicolumn{6}{|l|}{ Abdominal obesity ${ }^{d}$} \\
\hline Unadjusted & $3704 / 7337$ & 1.0 (Reference) & $1848 / 3559$ & 1.06 & $(0.98-1.15)$ \\
\hline Multivariable adjusted & & 1.0 (Reference) & & 1.06 & $(0.98-1.15)$ \\
\hline \multicolumn{6}{|l|}{ High fat mass index } \\
\hline Unadjusted & $3061 / 4846$ & 1.0 (Reference) & $1514 / 2467$ & 0.93 & $(0.84-1.02)$ \\
\hline Multivariable adjusted & & 1.0 (Reference) & & 1.03 & $(0.93-1.15)$ \\
\hline \multicolumn{6}{|l|}{ Diabetes mellitus ${ }^{f}$} \\
\hline Unadjusted & $363 / 8253$ & 1.0 (Reference) & $237 / 4155$ & 1.31 & $(1.11-1.56)$ \\
\hline Multivariable adjusted & & 1.0 (Reference) & & 1.27 & $(1.08-1.51)$ \\
\hline Multivariable adjusted a and BMI & & 1.0 (Reference) & & 1.25 & $(1.04-1.49)$ \\
\hline Multivariable adjusted a and FMI & & 1.0 (Reference) & & 1.25 & $(1.00-1.57)$ \\
\hline \multicolumn{6}{|l|}{ Cardiovascular disease $^{\mathrm{g}}$} \\
\hline Unadjusted & $134 / 8253$ & 1.0 (Reference) & $89 / 4155$ & 1.33 & $(1.01-1.74)$ \\
\hline Multivariable adjusted ${ }^{a}$ & & 1.0 (Reference) & & 1.31 & $(0.99-1.72)$ \\
\hline Multivariable adjusted ${ }^{\mathrm{a}}$ and $\mathrm{BMI}$ & & 1.0 (Reference) & & 1.41 & $(1.06-1.88)$ \\
\hline Multivariable adjusted ${ }^{\mathrm{a}}$ and $\mathrm{FMI}$ & & 1.0 (Reference) & & 1.13 & $(0.78-1.64)$ \\
\hline
\end{tabular}

$\mathrm{OR}=$ odds ratios; $\mathrm{Cl}=$ confidence intervals

${ }^{a}$ Adjusted for province, ethnicity, marital status, smoking and alcohol use.

${ }^{\mathrm{b}} \mathrm{High}$ level of physical activity

${ }^{c} \mathrm{BMI} \geq 30 \mathrm{~kg} / \mathrm{m}^{2}$

${ }^{\mathrm{d}}$ Waist circumference $\geq 102 \mathrm{~cm}$ for men and $\geq 88 \mathrm{~cm}$ for women

e Defined as fat mass index $\geq$ age and sex specific 75 percentiles(Schutz, Kyle, \& Pichard, 2002)

${ }^{f}$ Self-reported diabetes mellitus

sSelf-reported coronary heart disease or stroke 